\title{
Symmetric Runge-Kutta Methods with Higher Derivatives and Quadratic Extrapolation ${ }^{\star}$
}

\author{
Gennady Yu. Kulikov ${ }^{1}$, Ekaterina Yu. Khrustaleva ${ }^{2}$, \\ and Arkadi I. Merkulov \\ ${ }^{1}$ School of Computational and Applied Mathematics, \\ University of the Witwatersrand, Private Bag 3, \\ Wits 2050, Johannesburg, South Africa \\ ${ }^{2}$ Ulyanovsk State University, L. Tolstoy Str. 42, \\ 432970 Ulyanovsk, Russia \\ gkulikov@cam.wits.ac.za, shabalkina@mail.ru, \\ merkul@vda.ru
}

\begin{abstract}
In this paper we study the symmetry of Runge-Kutta methods with higher derivatives. We find conditions which provide this property for the above numerical methods. We prove that the family of E-methods constructed earlier consists of symmetric methods only, which lead to the quadratic extrapolation technique in practice.
\end{abstract}

\section{Introduction}

Here, we study Runge-Kutta methods with higher derivatives applied to ordinary differential equations (ODEs) of the form

$$
x^{\prime}(t)=g(t, x(t)), \quad t \in\left[t_{0}, t_{0}+T\right], \quad x\left(t_{0}\right)=x^{0}
$$

where $x(t) \in \mathbb{R}^{n}$ and $g: D \subset \mathbb{R}^{n+1} \rightarrow \mathbb{R}^{n}$ is a sufficiently smooth function. We remark that these numerical methods proved their efficiency. So, there are many papers published in this field (see [2], 3], 5], 7], 86, [11, [12, [13] and so on).

All methods of such sort can be represented as follows:

$$
\begin{gathered}
x_{k i}=x_{k}+\tau_{k} \sum_{j=1}^{l} \sum_{r=0}^{p_{j}} \tau_{k}^{r} a_{i j}^{(r)} g^{(r)}\left(t_{k j}, x_{k j}\right), \quad i=1,2, \ldots, l, \\
x_{k+1}=x_{k}+\tau_{k} \sum_{j=1}^{l} \sum_{r=0}^{p_{j}} \tau_{k}^{r} b_{j}^{(r)} g^{(r)}\left(t_{k j}, x_{k j}\right), \quad k=0,1, \ldots, K-1,
\end{gathered}
$$

where $x_{0}=x^{0}, t_{k j} \stackrel{\text { def }}{=} t_{k}+c_{j} \tau_{k}, g^{(r)}\left(t_{k j}, x_{k j}\right)$ denotes the $r$-th derivative 1 of the right-hand side of problem (1) with respect to $t$ evaluated at the point $\left(t_{k j}, x_{k j}\right)$,

\footnotetext{
* This work was supported in part by the National Research Foundation of South Africa under grant No. FA2004033000016.

${ }^{1}$ Here and below the zero-derivative implies the original function.
} 
the coefficients $a_{i j}^{(r)}, b_{j}^{(r)}$ and $c_{j}, i, j=1,2, \ldots, l, r=0,1, \ldots, p_{j}$ are real numbers $\left(c_{j} \in[0,1]\right)$, and $\tau_{k}$ is a step size which may be fixed or variable. In general, the numbers $p_{j}$ of the used derivatives are not equal.

When method (2) is implicit the important task is its correct implementation. In this case, we have to involve an additional iterative scheme and to keep a sufficient number of iteration steps, as indicated in [11, [12, in order to ensure high order convergence rate.

Here, we investigate the symmetry property of method (2). We find the conditions on the coefficients $a_{i j}^{(r)}, b_{j}^{(r)}$ and $c_{j}$ which guarantee that method (2) is symmetric. For example, these conditions are used to prove the symmetry of E-methods developed in [11], [12. Actually, the present study extends the results given in [4], 9] and [10] to the class of Runge-Kutta methods with higher derivatives. We just want to emphasize that symmetric numerical methods are of great importance when applied to reversible problems (see [6]) or when used as underlying methods in extrapolation algorithms (see the cited papers). However, we restrict ourselves to the quadratic extrapolation issue only in this paper.

\section{Symmetric Runge-Kutta Methods with Higher Derivatives}

It is clear that any particular Runge-Kutta method (2) with higher derivatives is determined uniquely by the set of coefficients $a_{i j}^{(r)}, b_{j}^{(r)}$ and $c_{j}$. Therefore, following Butcher's idea (see, for example, [1] or [4]), we represent method (2) by a partitioned tableau of the form

$$
\begin{array}{c|ccccccccc}
c_{1} & a_{11}^{(0)} & a_{11}^{(1)} & \ldots & a_{11}^{\left(p_{1}\right)} & \ldots & a_{1 l}^{(0)} & a_{1 l}^{(1)} & \ldots & a_{1 l}^{\left(p_{l}\right)} \\
c_{2} & a_{21}^{(0)} & a_{21}^{(1)} & \ldots & a_{21}^{\left(p_{1}\right)} & \ldots & a_{2 l}^{(0)} & a_{2 l}^{(1)} & \ldots & a_{2 l}^{\left(p_{l}\right)} \\
\vdots & \vdots & \vdots & \ddots & \vdots & \ddots & \vdots & \vdots & \ddots & \vdots \\
c_{l} & a_{l 1}^{(0)} & a_{l 1}^{(1)} & \ldots & a_{l 1}^{\left(p_{1}\right)} & \ldots & a_{l l}^{(0)} & a_{l l}^{(1)} & \ldots & a_{l l}^{\left(p_{l}\right)} \\
\hline & b_{1}^{(0)} & b_{1}^{(1)} & \ldots & b_{1}^{\left(p_{1}\right)} & \ldots & b_{l}^{(0)} & b_{l}^{(1)} & \ldots & b_{l}^{\left(p_{l}\right)}
\end{array}
$$

containing all the coefficients of a Runge-Kutta method with higher derivatives. The matrix $A$, consisting of the coefficients $a_{i j}^{(r)}$, is a real matrix of dimension $l \times\left(p_{1}+p_{2}+\ldots+p_{l}\right) l$, and the other coefficients form the real vectors $b$ and $c$ of dimensions $\left(p_{1}+p_{2}+\ldots+p_{l}\right) l$ and $l$, respectively, in tableau (3).

It is known that symmetric one-step methods are defined via their adjoint counterparts, as explained, for example, in [4, p. 219-222] or in [9]. Therefore our task now is to extend the appropriate results derived for coefficients of adjoint Runge-Kutta methods to the case of method (2) involving higher derivatives.

So, we start with

Theorem 1. Let method (2) be an l-stage Runge-Kutta formula with higher derivatives, whose coefficients are given by tableau (3). Then its adjoint method 
is equivalent to an l-stage Runge-Kutta formula with higher derivatives of the form (2) whose coefficients $a_{i j}^{(r)-}, b_{j}^{(r)-}$ and $c_{j}^{-}$satisfy

$$
\begin{gathered}
c_{j}^{-}=1-c_{l+1-j}, \\
a_{i j}^{(r)-}=(-1)^{r}\left(b_{l+1-j}^{(r)}-a_{l+1-i, l+1-j}^{(r)}\right), \\
b_{j}^{(r)-}=(-1)^{r} b_{l+1-j}^{(r)}, r=0,1, \ldots, p_{j}, i, j=1,2, \ldots, l .
\end{gathered}
$$

Proof. It is well-known (see the cited papers) that in order to find the adjoint Runge-Kutta method with higher derivatives we have to interchange the pair $\left(t_{k}, x_{k}\right)$ with the pair $\left(t_{k+1}, x_{k+1}\right)$ and to replace $\tau$ with $-\tau$ in the increment function of the method (2). It follows from the fact that method (2) belongs to the class of one-step methods.

Having fulfilled this transformation we arrive at an l-stage Runge-Kutta method of the form

$$
\begin{gathered}
x_{k i}=x_{k}+\tau_{k} \sum_{j=1}^{l} \sum_{r=0}^{p_{j}}(-1)^{r} \tau_{k}^{r}\left(b_{j}^{(r)}-a_{i j}^{(r)}\right) g^{(r)}\left(t_{k j}, x_{k j}\right), \quad i=1,2, \ldots, l, \\
x_{k+1}=x_{k}+\tau_{k} \sum_{j=1}^{l} \sum_{r=0}^{p_{j}}(-1)^{r} \tau_{k}^{r} b_{j}^{(r)} g^{(r)}\left(t_{k j}, x_{k j}\right), \quad k=0,1, \ldots, K-1,
\end{gathered}
$$

where $t_{k j}=t_{k}+\left(1-c_{j}\right) \tau_{k}$. If we now rearrange the nodes $c_{i}^{-}=1-c_{l+1-i}$ of method (5) to make them increasing; i.e., rearrange indexes by the rule $i \longleftrightarrow$ $l+1-i$ and $j \longleftrightarrow l+1-j$ we will yield the conventional Runge-Kutta method with higher derivatives of the form (2) whose coefficients $a_{i j}^{(r)-}, b_{j}^{(r)-}$ and $c_{j}^{-}$ satisfy formulas (4). The theorem is proved.

Papers 4, p. 219-222], 9] define a symmetric one-step method to be a method whose increment function coincides with the increment function of its adjoint method. Thus, Theorem 1 says that it is sufficient to require the following equalities:

$$
\begin{gathered}
c_{j}=1-c_{l+1-j}, \\
a_{i j}^{(r)}=(-1)^{r}\left(b_{l+1-j}^{(r)}-a_{l+1-i, l+1-j}^{(r)}\right), \\
b_{j}^{(r)}=(-1)^{r} b_{l+1-j}^{(r)}, r=0,1, \ldots, p_{j}, i, j=1,2, \ldots, l .
\end{gathered}
$$

Note that formulas (6) will be necessary for the symmetry if method (2) is irreducible (see [14]). It is also important to understand that conditions (6b), (6c) imply

$$
p_{j}=p_{l+1-j}, \quad j=1,2, \ldots, l ;
$$

i.e., equality (7) is required for any symmetric irreducible Runge-Kutta method with higher derivatives.

Our next goal is to show that symmetric irreducible Runge-Kutta methods of the form (2) do exist. 


\section{Symmetry of E-Methods with Higher Derivatives}

To approach this, we refer to the family of Runge-Kutta methods with higher derivatives constructed by the collocation technique with multiple nodes (see [11, 12]). They have been called E-methods with higher derivatives and are of the form

$$
\begin{gathered}
x_{k+1 / 2}=x_{k}+\tau_{k} \sum_{r=0}^{p} \tau_{k}^{r}\left(a_{1}^{(r)} g_{k}^{(r)}+a_{3}^{(r)} g_{k+1}^{(r)}\right)+\tau_{k} a_{2}^{(0)} g_{k+1 / 2}^{(0)}, \\
x_{k+1}=x_{k}+\tau_{k} \sum_{r=0}^{p} \tau_{k}^{r}\left(b_{1}^{(r)} g_{k}^{(r)}+b_{3}^{(r)} g_{k+1}^{(r)}\right)+\tau_{k} b_{2}^{(0)} g_{k+1 / 2}^{(0)}
\end{gathered}
$$

where $g_{k+i}^{(r)} \stackrel{\text { def }}{=} g^{(r)}\left(t_{k+i}, x_{k+i}\right), i=0,1 / 2,1$, and $p$ is the number of derivatives of the right-hand side of problem (1) used to compute the numerical solution. Evidently, method (8) belongs to the class of Runge-Kutta methods with higher derivatives, and its coefficients form the tableau

$$
\begin{array}{c|ccccccccc}
0 & 0 & 0 & \ldots & 0 & 0 & 0 & 0 & \ldots & 0 \\
1 / 2 & a_{1}^{(0)} & a_{1}^{(1)} & \ldots & a_{1}^{(p)} & a_{2}^{(0)} & a_{3}^{(0)} & a_{3}^{(1)} & \ldots & a_{3}^{(p)} \\
1 & b_{1}^{(0)} & b_{1}^{(1)} & \ldots & b_{1}^{(p)} & b_{2}^{(0)} & b_{3}^{(0)} & b_{3}^{(1)} & \ldots & b_{3}^{(p)} \\
\hline & b_{1}^{(0)} & b_{1}^{(1)} & \ldots & b_{1}^{(p)} & b_{2}^{(0)} & b_{3}^{(0)} & b_{3}^{(1)} & \ldots & b_{3}^{(p)}
\end{array}
$$

and satisfy

$$
\begin{gathered}
a_{1}^{(r)}=\frac{p+1}{r ! 2^{p+r+2}} \sum_{i=0}^{p-r} \sum_{l=0}^{i+r} \sum_{j=0}^{p+1} \frac{(-1)^{l}(i+r) !}{l !(i+r-l) ! j !(p+1-j) !(l+j+2)} \\
\times \sum_{q=0}^{i} \frac{(p+q) !}{q ! 2^{q}}, \quad r=0,1, \ldots, p, \\
a_{2}^{(0)}=\frac{(p+1) !}{2} \sum_{l=0}^{p+1} \frac{(-1)^{l}}{l !(p+1-l) !(2 l+1)}, \\
a_{3}^{(r)}=\frac{(-1)^{r+1}(p+1)}{r ! 2^{p+r+2}} \sum_{i=0}^{p-r} \sum_{l=0}^{i+r} \sum_{j=0}^{p+1} \frac{(-1)^{j}(i+r) !}{l !(i+r-l) ! j !(p+1-j) !(l+j+2)} \\
\times \sum_{q=0}^{i} \frac{(p+q) !}{q ! 2^{q}}, \quad r=0,1, \ldots, p, \\
b_{1}^{(r)}=a_{1}^{(r)}+(-1)^{r} a_{3}^{(r)}, \quad b_{2}^{(0)}=2 a_{2}^{(0)}, \quad b_{3}^{(r)}=(-1)^{r} a_{1}^{(r)}+a_{3}^{(r)} .
\end{gathered}
$$

It was proven in 12 that method (8) with the coefficients calculated by formulas (10) is $A$-stable for any $p$. Thus, the E-methods with higher derivatives are a good means for a practical implementation when differentiation of the right-hand side of ODE (1) is not too complicated. Now we prove the symmetry of method (8). 
Theorem 2. E-method (8) with coefficients (10) is symmetric for any integer $p \geq 0$.

Proof. To prove this theorem, it is sufficient to check the symmetry conditions $(6),(7)$ for the coefficients in tableau (9) satisfying formulas (10) (see Section 2). It is obvious that $(6 \mathrm{a})$ and $(7)$ hold. Then, by calculating the right-hand side of formula (6b) applied to the method (8) and using (10d), we obtain the following:

$$
\begin{aligned}
& (-1)^{r}\left(b_{3}^{(r)}-a_{3}^{(r)}\right)=(-1)^{r}\left((-1)^{r} a_{1}^{(r)}+a_{3}^{(r)}-a_{3}^{(r)}\right)=a_{1}^{(r)}, \\
& (-1)^{r}\left(b_{2}^{(0)}-a_{2}^{(0)}\right)=(-1)^{r}\left((-1)^{r} a_{2}^{(0)}+a_{2}^{(0)}-a_{2}^{(0)}\right)=a_{2}^{(0)}, \\
& (-1)^{r}\left(b_{1}^{(r)}-a_{1}^{(r)}\right)=(-1)^{r}\left((-1)^{r} a_{3}^{(r)}+a_{1}^{(r)}-a_{1}^{(r)}\right)=a_{3}^{(r)} .
\end{aligned}
$$

The condition $(6 \mathrm{~b})$ also holds. In the same way, we easily check the last condition (6c). Theorem 2 is proved.

Next, we confirm this theoretical result with the numerical examples below which show that method (8) with coefficients (10) provide quadratic extrapolation for different values of the parameter $p$. We refer the reader to [4], 10] for particulars of quadratic extrapolation.

\section{Quadratic Extrapolation}

First, we apply formulas (10) to calculate coefficients of the following E-methods of orders 6 and 8 , respectively; i.e., when $p=1$ and $p=2$ :

$$
\begin{gathered}
x_{k+1 / 2}=x_{k}+\frac{131}{480} \tau_{k} g_{k}^{(0)}+\frac{23}{960} \tau_{k}^{2} g_{k}^{(1)}-\frac{19}{480} \tau_{k} g_{k+1}^{(0)}+\frac{7}{960} \tau_{k}^{2} g_{k+1}^{(1)}+\frac{4}{15} \tau_{k} g_{k+1 / 2}^{(0)}, \\
x_{k+1}=x_{k}+\frac{7}{30} \tau_{k}\left(g_{k}^{(0)}+g_{k+1}^{(0)}\right)+\frac{1}{60} \tau_{k}^{2}\left(g_{k}^{(1)}-g_{k+1}^{(1)}\right)+\frac{8}{15} \tau_{k} g_{k+1 / 2}^{(0)} \\
x_{k+1 / 2}=x_{k}+\frac{689}{2240} \tau_{k} g_{k}^{(0)}+\frac{169}{4480} \tau_{k}^{2} g_{k}^{(1)}+\frac{17}{8960} \tau_{k}^{3} g_{k}^{(2)}-\frac{81}{2240} \tau_{k} g_{k+1}^{(0)} \\
+\frac{41}{4480} \tau_{k}^{2} g_{k+1}^{(1)}-\frac{19}{26880} \tau_{k}^{3} g_{k+1}^{(2)}+\frac{8}{35} \tau_{k} g_{k+1 / 2}^{(0)} \\
x_{k+1}=x_{k}+\frac{57}{210} \tau_{k}\left(g_{k}^{(0)}+g_{k+1}^{(0)}\right)+\frac{1}{35} \tau_{k}^{2}\left(g_{k}^{(1)}-g_{k+1}^{(1)}\right) \\
+\frac{1}{840} \tau_{k}^{3}\left(g_{k}^{(2)}+g_{k+1}^{(2)}\right)+\frac{16}{35} \tau_{k} g_{k+1 / 2}^{(0)} .
\end{gathered}
$$

Second, we test E-methods (11) and (12) on the problem

$$
\begin{gathered}
x_{1}^{\prime}(t)=2 t x_{2}(t)^{1 / 5} x_{4}(t), \quad x_{2}^{\prime}(t)=10 t \exp \left\{5\left(x_{3}(t)-1\right)\right\} x_{4}(t), \\
x_{3}^{\prime}(t)=2 t x_{4}(t), \quad x_{4}^{\prime}(t)=-2 t \ln \left\{x_{1}(t)\right\}
\end{gathered}
$$

where $x_{i}(0)=1, i=1,2,3,4$. Problem (13) has the following exact solution: $x_{1}(t)=\exp \left\{\sin \left(t^{2}\right)\right\}, x_{2}(t)=\exp \left\{5 \sin \left(t^{2}\right)\right\}, x_{3}(t)=\sin \left(t^{2}\right)+1, x_{4}(t)=\cos \left(t^{2}\right)$. 
Table 1. Global errors of the E-method (11) with $q$ extrapolations applied to (13)

\begin{tabular}{c|ccccc}
\hline & & \multicolumn{5}{|c}{ fixed step size } \\
$q$ & $\tau=1.00 \cdot 10^{-1}$ & $\tau=6.67 \cdot 10^{-2}$ & $\tau=4.44 \cdot 10^{-2}$ & $\tau=2.96 \cdot 10^{-2}$ & $\tau=1.98 \cdot 10^{-2}$ \\
\hline 0 & $1.1822 \cdot 10^{-01}$ & $1.2221 \cdot 10^{-02}$ & $1.0970 \cdot 10^{-03}$ & $9.6250 \cdot 10^{-05}$ & $8.4474 \cdot 10^{-06}$ \\
1 & $1.3139 \cdot 10^{-04}$ & $4.1088 \cdot 10^{-06}$ & $1.2647 \cdot 10^{-07}$ & $5.1884 \cdot 10^{-09}$ & $2.1093 \cdot 10^{-10}$ \\
2 & $1.8188 \cdot 10^{-06}$ & $1.4897 \cdot 10^{-08}$ & $2.0497 \cdot 10^{-10}$ & $3.9575 \cdot 10^{-12}$ & $3.6386 \cdot 10^{-13}$ \\
\hline
\end{tabular}

Table 2. Global errors of the E-method (12) with $q$ extrapolations applied to (13)

\begin{tabular}{c|ccccc}
\hline & & \multicolumn{5}{|c}{ fixed step size } \\
$q$ & $\tau=1.00 \cdot 10^{-1}$ & $\tau=6.67 \cdot 10^{-2}$ & $\tau=4.44 \cdot 10^{-2}$ & $\tau=2.96 \cdot 10^{-2}$ & $\tau=1.98 \cdot 10^{-2}$ \\
\hline 0 & $2.2594 \cdot 10^{-03}$ & $7.9717 \cdot 10^{-05}$ & $2.9465 \cdot 10^{-06}$ & $1.1041 \cdot 10^{-07}$ & $4.4174 \cdot 10^{-09}$ \\
1 & $3.1320 \cdot 10^{-06}$ & $2.8233 \cdot 10^{-08}$ & $3.1639 \cdot 10^{-10}$ & $6.1941 \cdot 10^{-12}$ & $2.4070 \cdot 10^{-13}$ \\
2 & $2.2455 \cdot 10^{-08}$ & $5.6611 \cdot 10^{-11}$ & $6.6482 \cdot 10^{-13}$ & $3.5671 \cdot 10^{-13}$ & $1.4103 \cdot 10^{-13}$ \\
\hline
\end{tabular}

Our aim in this section is to check that E-methods (11) and (12) are symmetric and, in fact, provide quadratic extrapolation in practice. To do this, we perform numerical integrations of problem (13) with 5 different fixed step sizes $\tau_{k}=\tau$ on the interval [0,3], as indicated in Tables 11 and 2, We use these methods without extrapolation $(q=0)$, with one extrapolation step $(q=1)$ and with two extrapolations $(q=2)$ as well. However, the underlying methods are implicit. Therefore we apply the Newton iteration with the trivial predictor in order to obtain the required numerical solution and keep the number of iteration steps per grid point high enough to ensure that the extrapolation works properly (see [10] for more detail). We choose integers $1,2,3$ and so on as the extrapolation sequence. The exact solution mentioned above is used to calculate the global errors of the integrations in the sup-norm and, hence, to find the actual order of the extrapolation algorithms.

Tables 1 and 2 exhibit clearly that the quadratic extrapolation definitely works with E-methods (11) and (12); i.e., each step of the extrapolation algorithm raises the accuracy of the underlying method by two orders. This experiment confirms also that both E-methods are symmetric and the theoretical results given above are correct.

\section{Conclusion}

In this paper we have discussed the symmetry property of Runge-Kutta methods with higher derivatives. We have found the coefficients of adjoint Runge-Kutta methods with higher derivatives (Theorem 1) and determined the general conditions on the coefficients of the methods under consideration to be symmetric (formulas $(6),(7))$. The new result is an extension of the well-known formulas for conventional Runge-Kutta methods (see the cited papers) and, of course, covers this case. Finally, we have given some examples of symmetric Runge-Kutta 
methods with higher derivatives and confirmed this property with practical computations. From this point of view, the family of E-methods derived earlier in 11] and [12 looks attractive if the cost of the derivative calculation is not too high. However, more experiments are needed to identify practical properties of the E-methods in detail.

\section{References}

1. Butcher, J.C.: Numerical methods for ordinary differential equations. John Wiley and Son, Chichester, 2003

2. Fehlberg, E.: Eine methode zur fehlerverkleinerung bein Runge-Kutta verfahren. ZAMM. 38 (1958) 421-426

3. Fehlberg, E.: New high-order Runge-Kutta formulas with step size control for systems of first and second order differential equations. ZAMM. 44 (1964) T17-T19

4. Hairer, E., Nørsett, S.P., Wanner, G.: Solving ordinary differential equations I: Nonstiff problems. Springer-Verlag, Berlin, 1993

5. Hairer, E., Wanner, G.: Solving ordinary differential equations II: Stiff and differential-algebraic problems. Springer-Verlag, Berlin, 1996

6. Hairer, E., Wanner, G., Lubich, C.: Geometric numerical integration: structure preserving algorithms for ordinary differential equations. Springer-Verlag, Berlin, 2002

7. Kastlunger, K.H., Wanner, G.: Runge-Kutta processes with multiple nodes. Computing. 9 (1972) 9-24

8. Kastlunger, K.H., Wanner, G.: On Turan type implicit Runge-Kutta methods. Computing. 9 (1972) 317-325

9. Kulikov, G.Yu.: Revision of the theory of symmetric one-step methods for ordinary differential equations, Korean J. Comput. Appl. Math., 5 (1998) No. 3, 289-318

10. Kulikov, G.Yu.: On implicit extrapolation methods for ordinary differential equations, Russian J. Numer. Anal. Math. Modelling., 17 (2002) No. 1, 41-69

11. Kulikov, G.Yu., Merkulov, A.I., Khrustaleva, E.Yu.: On a family of A-stable collocation methods with high derivatives. In: Marian Bubak, Geert Dick van Albada, Peter M. A. Sloot, and Jack J. Dongarra (eds.): Computational Science ICCS 2004. 4th International Conference, Krakow, Poland, June 6-9 2004. Proceedings, Part II. Lecture Notes in Computer Science, 3037 (2004) 73-80

12. Kulikov, G.Yu., Merkulov, A.I.: On one-step collocation methods with higher derivatives for solving ordinary differential equations. (in Russian) Zh. Vychisl. Mat. Mat. Fiz. 44 (2004) No. 10, 1782-1807; translation in Comput. Math. Math. Phys. 44 (2004) No. 10, 1696-1720

13. Nørsett, S.P.: One-step methods of Hermite type for numerical integration of stiff systems. BIT. 14 (1974) 63-77

14. Stetter, H.J.: Analysis of discretization methods for ordinary differential equations, Springer-Verlag, Berlin, 1973 